\title{
Electronic and magnetic properties of the Fe-doped $\mathrm{CuInS}_{2}$
}

\author{
C. Tablero \\ Instituto de Energía Solar, Universidad Politécrnica de Madizi, Cindad Universitaria s/n, 28040 Madizi, Spain
}

\section{A R T I C L E I N F O}

\section{Introduction}

Considerable interest has been shown in the chalcopyrite compounds as a result of their important technological applications in nonlinear optics, light-emitting diodes, and solar cells [1]. $\mathrm{Cu}\left(\mathrm{Ag},[\mathrm{n}, \mathrm{Ga}) \mathrm{X}_{2}\right.$ systems have recently attracted much attention [2], because these materials have direct band gaps in the range desirable for applications in solid state lighting and high-efficiency solar cells. It can be grown using thin film technology and is one promising solutions for the production of photovoltaic energy [3]. In particular, CulnS $S_{2}$ is a promising absorbing material for the production of high-efficiency solar cells, because of its well suited band gap and low preparation cost of polycrystalline thin films.

Chalcopyrite type $\left[-[][-V]_{2}\right.$ compounds are considered capable of containing transition elements, since the chalcopyrite mineral (antiferromagnetic $\mathrm{CuFeS}_{2}$ ) has Fe as its constituent element. Transition atom impurities have been known to introduce deep levels into the band gaps of semiconductors, which not only control a concentration and a type of conducting carrier but also act as activators [4] or killers of luminescence. [n recent years, chalcopyrites and other compounds doped with transition atoms are attracting interest as magnetic semiconductors for next-generation spinelectronics device applications [5-7].

Experimentally, the optical absorption spectra of single $\mathrm{CulnS}_{2}$ crystals doped with $\mathrm{Fe}$, where the In atom is substituted by $\mathrm{Fe}$, present a sub-gap absorption band with a threshold at $0.8 \mathrm{eV}$ [8]. This absorption band has been associated with the photoionization transition from the valence band (VB) to the unoccupied impurityrelated deep level.

Thus it is interesting to analyze the electronic and magnetic properties of $\mathrm{Fe}$ doping in the copper indium sulfide chalcopyrite and to compare it with the experimental results in the literature.

E-mail address: ctablero@etsit.upm.es

\section{Calculations}

In order to obtain the electronic properties, we have used the density-functional theory (DFT) [9]. The standard Kohn-Sham (KS) [10] equations are solved self-consistently [11]. For the exchange and correlation term, the local-spin density approximation (LDA) has been used with the Perdew-Zunger parametrization to the Ceperley-Alder numerical data [12], and the generalized gradjent approximation (GGA) in the form of Perdew et al. [13]. The standard Troullier-Martins [14] pseudopotential is adopted and expressed in the Kleinman-Bylander [15] factorization. The KS orbitals are represented by using a linear combination of confined pseudoatomic orbitals [16]. An analysis of the basis set has been carried out using from single-zeta to double-zeta with polarization basis sets for all atoms and varying the number of the special $k$ points in the irreducible Brillouin zone. We have used 16-, 32and 64-atom supercells. In all of the results presented in this work a double-zeta with polarization functions basis set has been used with periodic boundary conditions with 64-atom supercells.

Because of the electronic properties of the systems analyzed, with an IB, the local correlation effects could be very important. The gap underestimation is also well-known when LDA and GGA are used. Therefore, a more profound study of these materials with respect to the correlation effect is carried out. We use the LDA/ GGA + U method described in Ref. [17] in order to improve the description of the states of the IB.

\section{Results and discussion}

The copper indium sulfide CulnS $S_{2}$ chalcopyrite have tetragonal structure obtained from the cubic ll-VI structure by occupying the group-II atom sites alternatively with group-] (Cu) and group-]II (In) atoms. Each anion (S atom) is coordinated by four cations (two $\mathrm{Cu}$ and two In atoms), whereas each cation is tetrahedrally surrounded by four anions. The experimental lattice 
parameters are: $a=5.52 \AA, c / 2 a=1.006$ and $u=0.214[18,19]$, the experimental gap is $1.48-1.55 \mathrm{eV}[20]$, and the In-S distance is $2.34 \AA$.

With our calculations, the gap obtained for the host chalcopyrite $\mathrm{CuInS}_{2}$ is 0.9 and $0.6 \mathrm{eV}$ with GGA and LDA, respectively. These values are lower than the experimental ones (1.48-1.55 eV [20]) because of the GGA/LDA gap underestimation. The gaps in the literature obtained with different methodologies, basis sets and different exchange-correlation functionals are: $-0.14 \mathrm{eV}$ [21], $0.01 \mathrm{eV}$ [22], $0.812 \mathrm{eV}$ [23], approximately zero [24], and $0.02 \mathrm{eV}$ [25]. Therefore, the results compare well with others in the literature. We later show that by using GGA/LDA + U, the gaps increase to 1.55 and $1.50 \mathrm{eV}$, respectively, which compare well with the experimental ones.

The Fe substitution by In ( $\mathrm{Fe}_{\text {In }}$ substitution) distorts the host structure. The relaxation of both the atomic positions and the lattice constants without symmetry constraints has been carried out for smaller cells. The relaxation has been considered accomplished when the forces on the atoms were lower than $0.004 \mathrm{eV}^{-1}$. After relaxing the lattice parameters and all atoms in the cell, the new lattice parameters are $a=5.528 \AA$ and $c / 2 a=0.979$, and the four distances Fe-S are different: $2.34,2.29,2.33$ and $2.31 \AA$.

We have carried out first principles calculations for the ferromagnetic (FM) and antiferromagnetic (AFM) Fe-doped CuInS $\mathrm{S}_{2}$ chalcopyrite using both, LDA and GGA. The energy differences per Fe atom between the AFM and FM configurations are -0.01 and
$0.02 \mathrm{eV}$. These results are similar to those obtained in Ref. [7] for the Fe-doped CuInSe 2 : from -0.02 to $+0.02 \mathrm{eV}$. At lower Fe concentration, both FM and AFM states have close energies and may coexist. Thus a mix of both AFM and FM states could be expected, i.e. no ordering of the magnetic moments is present.

One of the main effects of substituting In host atoms by Fe in the electronic structure of the host chalcopyrite is to generate a band (IB) above the VB fairly close to the conduction band (CB). Increasing the Fe concentration creates more and more new levels above the VB. For the FM state only an IB with spin-down character is present, whereas for the AFM state, both spin characteristics are present due to the symmetry of the up and down components. The Fermi energy is between the VB and the IB indicating that the IB is empty. Therefore this IB has an acceptor character. Nevertheless, because of the LDA/GGA gap underestimation, the IB band overlaps with the $\mathrm{CB}$.

A study of the density of states (DOS) indicate that this IB is made up by the $d$-Fe orbitals. In order to analyze the structure of this IB the projected DOS on the $d$-Fe orbitals corresponding to one Fe atom with anti parallel alignment is represented in Figure 1 for the FM and AFM components, and with LDA and GGA. For the AFM alignment, half of the Fe atoms have a projected DOS with opposed sign to that shown in the figure. A band between $0.55-$ $1.3 \mathrm{eV}$ above the $\mathrm{VB}$ is present for the FM and AFM alignment for both, GGA and LDA. These values compare well with the experimental ones [8] ( $0.8 \mathrm{eV}$ above VB). The lower part (in energy) of
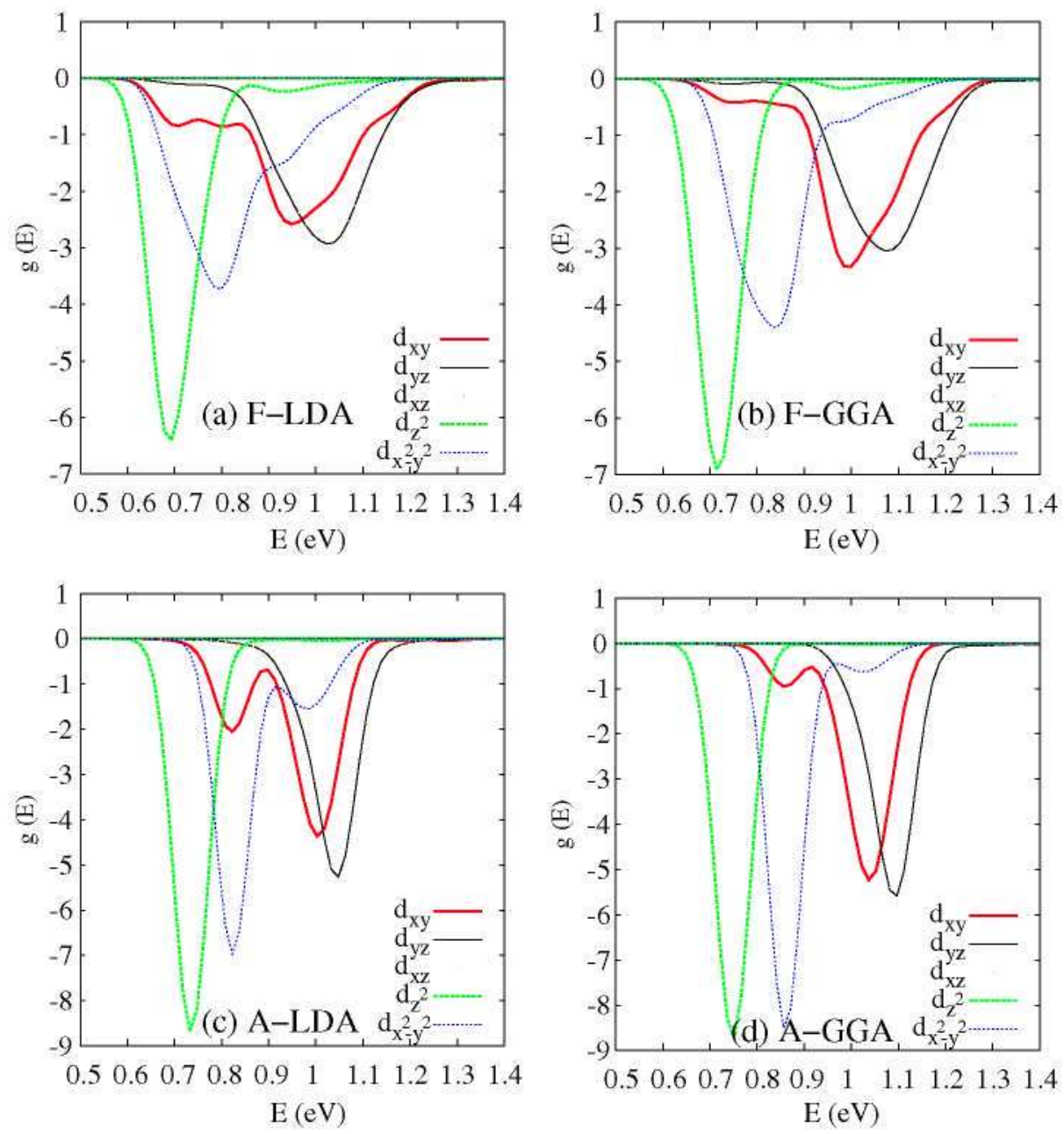

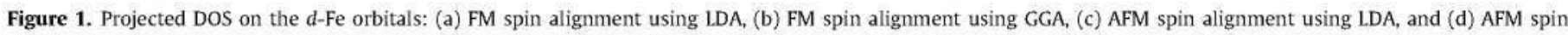
alignment using GGA. The VB top energy has been chosen as zero energy in the figure. 
this band is made up of the $d_{z^{2}}$ and the $d_{x^{2}-y^{2}}$-Fe orbitals, whereas the component with larger energy is made up of the $d_{x y^{-}}, d_{x z^{-}}$and $d_{y z}$-Fe orbitals.

Additionally, the number of states $(\eta)$ with energy lower than $E$, i.e. the integrated DOS, is plotted in Figure 2 for the majority-and minority-spin channels. Note that for the AFM order there are Fe atoms with the spin components exchanged in respect to the figure. The panels (a) and (b) correspond to the total and Fe integrated DOS. The GGA and LDA, and the FM and AFM results in Figure 2 are very similar and almost indistinguishable.

For the $\mathrm{Fe}_{\ln }$ substitution each $\mathrm{Fe}$ impurity atom adds five additional electrons to the majority-spin VB. The total magnetization of the unit cell is $5 \mu_{\mathrm{B}}$ per Fe atom for the FM order. The number of spin-down valence states (and electrons) is not changed by the Fe impurity with respect to the number of VB spin-down states of the host chalcopyrite (Figure 2a). Therefore, the total number of valence electrons for the minority-spin component is half of the total number of valence electrons in the host chalcopyrite.

The Fe charge of the two spin components at the VB are $q_{\mathrm{VB}}^{(+)}\left(\mathrm{Fe}_{\mathrm{In}}\right)=5.9$ and $q_{\mathrm{VB}}^{(-)}\left(\mathrm{Fe}_{\mathrm{In}}\right)=1.8$ electrons, respectively (Figure $2 \mathrm{~b})$. These populations correspond to the number of states of the Fe atom for the Fermi energy. The Fermi energy is between the VB top (zero energy) and the IB in Figure 2. As the acceptor $d$-Fe IB is above the Fermi energy, these bands have no charge. Thus the total Fe charge is that corresponding to the VB charge. In fact, a population analysis using the Mulliken and Löwdin schemes leads to similar values for the atomic charges of the Fe with LDA and GGA. According to the Mulliken and Löwdin schemes the Fe atom has 5.9 electrons for the spin-up component and 1.8 electrons for the spin-down.

From the previous results, the total magnetic moment of the cell is not located strictly on the Fe atoms. Indeed, the magnetic moment of the Fe atom is $\sim 4.1-4.2 \mu_{\mathrm{B}}$, whereas the total magnetic moment of the cell is $5 \mu_{\mathrm{B}}$ per Fe atom for the FM order. The Fe atom induces small negative moments on the first-nearest neighbor $\mathrm{S}$ atoms, which have a local magnetic moment of $\sim 0.1 \mu_{\mathrm{B}}$. The other atoms carry much smaller magnetic moments $\left(1.3 \mu_{\mathrm{B}}\right.$ between 59 atoms for the 64-atom supercell) and can be ignored.

In order to correct the level positions for isolated defects, it is usually assumed [26] that the energy levels of the acceptor-like de-
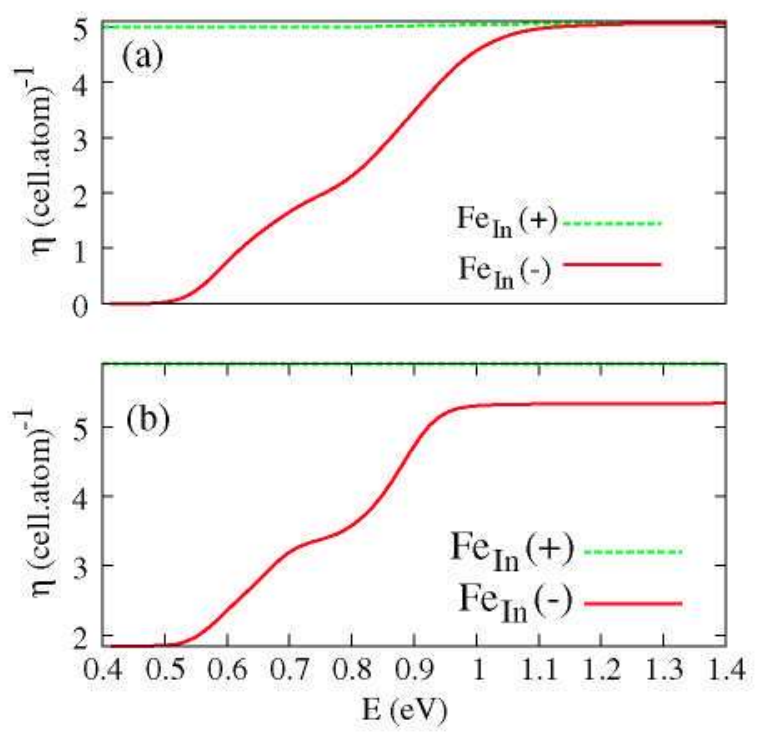

Figure 2. Total (a), and Fe (b) integrated DOS $\eta$ per Fe atom for the spin-up (+) and spin-down (-) alignments. The zero origin in the energy axis is the VB top energy. For the total integrated DOS (panel a), the $\eta$ axis origin is the number of states of the host chalcopyrite at the VB top.
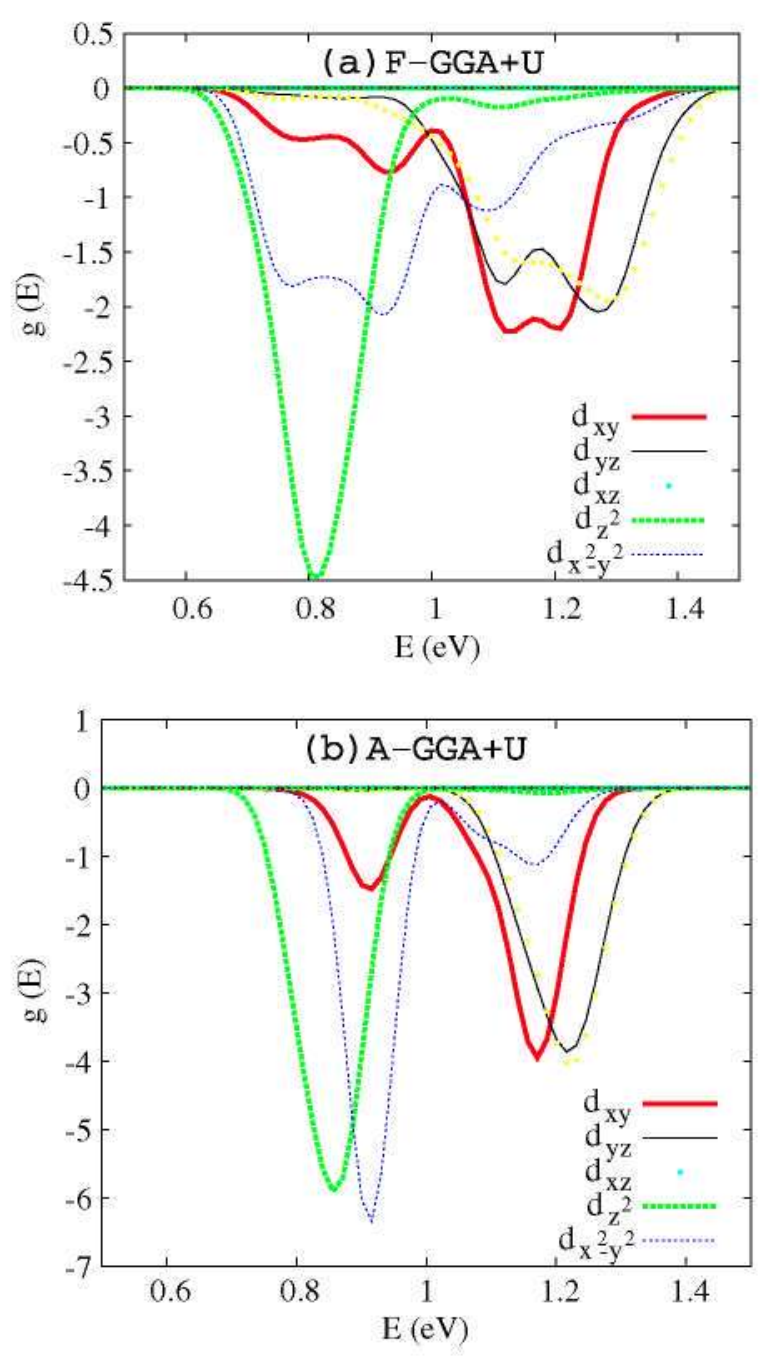

Figure 3. Projected DOS on the $d$-Fe orbitals using GGA + U with (a) FM spin alignment, and (b) AFM spin alignment. The VB top energy has been chosen as zero energy in the figure.

fects follow the VB edge and are thus unchanged while donor-like defect levels follow the edge of the $\mathrm{CB}$ and are thus shifted upward by the same amount as the band gap. Therefore, for the $\mathrm{Fe}_{\text {In }}$ substitution the states associated with Fe would not be affected for the well-known gap underestimation and they would have similar energies with respect to the states of the VB.

In order to verify the previous assumption for this case, we have carried out the calculations using the GGA/LDA + U formalism described in Ref. [17] with $U=5 \mathrm{eV}$. The gaps obtained with GGA $+U$ and $L D A+U$ are 1.55 and $1.50 \mathrm{eV}$, respectively, which compare well with the experimental ones $(1.48-1.55 \mathrm{eV})$. The Fe states in the gap present small modification (Figure 3 ) compared with the GGA/LDA (Figure 1). The AFM and FM energy difference per Fe atom $(-0.02 \mathrm{eV})$ is also similar to the GGA/LDA. Therefore, the GGA/LDA reproduces the position of the acceptor $d$-Fe band reasonably well for the $\mathrm{Fe}_{\mathrm{In}}$ substitution and the AFM and FM energy difference. In addition, the GGA/LDA + U locate the $d$-Fe band and the gap more closely to experimental results [8].

\section{Conclusions}

In summary, we report the results of the electronic and magnetic properties of the Fe-doped chalcopyrite $\mathrm{CuInS}_{2}$ using first principles GGA/LDA and GGA/LDA + U calculations. The calcula- 
tions have revealed structures of different complexity. In both cases an IB made up of the $d$-Fe orbitals is present in the gap. $\mathrm{The}_{\mathrm{Fe}_{\mathrm{ln}}}$ substitution behaves like an acceptor with an IB empty in the gap above $0.8-1.0 \mathrm{eV}$ from the VB. These results agree very well with the experimental results. In particular, with the experimental photoionization transition from the VB to the acceptor-related Fe deep level. The FM and AFM states have close energies and may coexist.

The main effect of GGA/LDA + $U$ is to remove the gap underestimation of the GGA/LDA. Although GGA/LDA results place the $d-F e$ band in agreement with experimental results, the GGA/LDA + U still locate it next to the experimental results.

\section{Acknowledgements}

This work has been supported by the GENESIS FV project of the National Spanish program CONSOLIDER (CSD2006-0004), by the European Commission through the funding of the project IBPOWER (Ref. N: Grant Agreement 211640), and by La Comunidad de Madrid through the funding of the project NUMANClA-2 (Ref. N: S2009/ENE-1477).

\section{References}

[1] J.L. Shay, J.H. Wernick, Ternary Chalcopyrite Semiconductors: Growth, Electronic Properties and Applications, Pergamon Press, Oxford, 1975; M.C. Ohmer, R. Randey, B.H. Bairamov, Mater. Res. Bull. 23 (1998) 16; R.W. Birkmire, E. Eser, Annu. Rev, Mater. Sci. 27 (1997) 625,

S. Chichibu, S. Shirakata, S. Jsomura, H. Nakanishi, Jpn. J. Appl. Phys. Part 136 (1997) 1703;

5.N. Rashkeev, W.R.L lambrecht, Phys. Rev. B 63 (2001) 165212.

[2] R.R. Reddy, K. Rama Gopal, K. Narasimhulu, L. Siva Sankara Reddy, K. Raghavendra Kumar, G. Balakrishnaiah, M. Ravi Kumar. J. Alloys Compd. 473 (2009) 28 ;

S.F. Chichibu, T. Ohmori, N. Shibata, T. Koyama, T. Onuma, J. Phys. Chem. Solids 66 (2005) 1868;

T.J. Coutts, K.A. Emery. J.S. Ward, Prog. Photovoltaics 10 (2002) 195;

T.J. Coutts, J.5. Ward, D.L. Young, K.A. Emery. T.A. Gessert, R. Noufi, Prog. Photovoltaics 11 (2003) 359.
|3| K. Zweibel, Prog. Photovoltaics 3 (1995) 279; B. Dimmler, H.W. Schock, Prog. Photovoltaics 4 (1996) 425; F. Karg, H. Aulich, W. Riedl, in: H.A. Ossenbrink, P. Helm, H. Ehmann (Eds.). Proceedings of the 14th European Photovoltaic Solar' Energy Conference, Barcelona, H.S. Stephens and Associates, Bedford, UK, 1997.

|4| C. Tablero. Physica B 404 (2009) 4023: A. Luque, A. Martí, E. Antolín, C. Tablero, Physica B 382 (2006) 320.

[5] T. DietI, H. Ohno, F. Matsukura, J. Cibert, D. Ferrand, Science 287 (2000) 1019 G. Sclımidt, D. Ferrand, L.W. Molenkamp. A.T. Filip. B.J. van Wees, Plyss. Rev. B 62 (2000) R4790;

1. Zutiæ. J. Fabian, 5. Das Sarma, Rev. Mod. Phys. 76 (2004) 323.

|6| C. Tablero. D. Fuertes Marrón, J. Phys. Chem. C 114 (2010) 2756: C. Tablero, J. Appl. Phys. 106 (2009) 073718.

|7| J.M. Raulot, C. Domain, J. Guillemoles, Plyss. Rev, B 71 (2005) 035203.

[8] K. Sato, M. Jsawa, N. Takahashi, H. Tsunoda, Jpn. J. Appl. Phys. 27 (1988) 1359.

|9| P. Hohenberg. W. Kohn, Plyys. Rev. B 136 (1964) 864

[10] W. Koln, L.J. Sham, Phys, Rev. 140 (1965) A1133.

[11] J.M. Soler, E. Artacho, J.D. Gale, A. García, J. Junquera, P. Ordejon, D. SánchezPortal. J. Phys. Condens. Matter 14 (2002) 2745 (and references therein).

[12] J.P. Perdew, A. Zunger, Phys. Rev. B 23 (1981) 5048; D.M. Ceperley, B.J. Alder, Phys. Rev. lett. 45 (1980) 566.

[13] J.P. Perdew, K. Burke, M. Ernzerhof, Phys. Rev, lett. 77 (1996) 3865; J.P. Perdew, K. Burke, M. Ernzerhof, Phys. Rev. lett. 78 (1997) 1396.

[14] N. Troullier, J.L. Martins, Phys. Rev. B 43 (1991) 1993.

[15] L. Kleinman, D.M. Bylander, Phys. Rev, Lett. 48 (1982) 1425; D.M. Bylander, L. Kleinman, Phys. Rev. B 41 (1990) 907.

[16] O.F. Sankey, D.J. Niklewski, Phys. Rev. B 40 (1989) 3979.

[17] C. Tablero, J. Chem. Phys. 130 (2009) 054903; C. Tablero. J. Plyss: Condens. Matter $20(2008) 325205$

[18] J.E. Jaffe, Alex Zunger, Phys. Rev. B 28 (1983) 5822.

[19] 0 . Madelung (Ed.), Semiconductors: Data Handbook, third edn., Springer, Berlin, 2004

[20] J.L. Shay, J.H. Wernick, Temary Chalcopyrite Semiconductors: Growth, Electronic properties, and applications, Pergamon Press, Oxford, 1975; B.R. Pamplin, T. Kiyosawa, K. Mastumoto, Prog. Cryst. Growth Charact. 1 (1979) 331;

B. Tell. J.L. Shay. H.M. Kasper, Phys. Rev. B 4 (1971) 2463; J. Gonzalez, C. Rincon, J. Appl. Phys. 65 (1989) 2031.

[21] J.E. Jaffe, Alex Zunger, Phys. Rev, B 27 (1983) 5176.

[22] S.N. Rashkeev, W.R.L. Lambrecht, Phys. Rev. B 63 (2001) 165212.

[23] A.H. Reshak, S. Auluck, PMC Phys. B 1 (2008) 12, doi: 10.1186/1754-042.

[24] M.G. Brik, J. Plyys.: Condens. Matter 21 (2009) 485502.

[25] J. tazewski, P.T. Jochym, K. Parlinski, J. Chem. Phys. 117 (2002) 2726

[26] 5.B. Zlang. 5.H. Wei, A. Zunger, H. Katayama-Yoshida, Phys. Rev, B 57 (1998) 9642 . 